\title{
Mathematical model for malaria with mosquito-dependent coefficient for human population with exposed class
}

\author{
Ram Singh ${ }^{1}$, Shoket Ali ${ }^{*}$, Madhu Jain ${ }^{3}$ and Ather Aziz Raina ${ }^{4}$ \\ ${ }^{1}$ Department of Mathematical Sciences, Baba Ghulam Shah Badshah, University, Rajouri, India. \\ ${ }^{2}$ Department of Mathematics, Lovely Professional University, Jalandhar, Punjab, India. \\ ${ }^{3}$ Department of Mathematics, Indian Institute of Technology Roorkee, India. \\ ${ }^{4}$ Department of Mathematics, Govt. Post Graduate College, Rajouri, India.
}

Submitted:16 March 2018; Revised: 17 June 2018; Accepted:17 August 2018

\begin{abstract}
In this paper, a SEIR (Susceptible, Exposed, Infectious and Recovered) mathematical model of transmission dynamics of malaria was proposed. In the model, mosquito population acts as the vector population and depends upon the human population for its growth and survival. It was shown that the environmental factors are conducive to the spread of malaria disease. It was also found that effective control programming against the spread of malaria is helpful in reducing the transmission dynamics of the disease. Sensitivity analysis was performed to show that spraying of pesticides and proper drainage system will effectively control the disease.
\end{abstract}

Keywords: Human and vector population, stability analysis, transmission dynamics.

\section{INTRODUCTION}

Malaria has become a worldwide problem and the worst affected countries are mostly African and Asian. Approximately 3 billion people have been affected in 109 countries. Every year there are approximately 250 million cases of malaria reported, which result in one million deaths (WHO report 2013).

Ross (1916) gave the first mathematical model of malaria transmission and several mathematical models have been formulated to investigate the transmission dynamics of malaria (Gupta et al., 1994; Hethcote, 2000; Ngwa \& Shu, 2000; Ngwa, 2004; Chitnis et al., 2006; 2012; Chavez et al., 2008; Ruan et al., 2008; Chamchod \&
Britton, 2011; Li, 2011; Erin et al., 2013; Xiao et al., 2013). Ngwa and Shu (2000) analysed a deterministic differential equation model for endemic malaria involving variable human and mosquito populations. Ngwa (2004) provided a solution by analysing the problem through the mathematical model. In the study the sample mathematical model has been considered and used a perturbation analysis and found that the death rate is nonzero, small and significant. Chitnis et al. (2006; 2012) gave a model for infectious agents where they found the effect of immigration and disease induced death rates. The study of malaria transmission dynamics through mathematical modelling is a vast and fascinating area wherein the developed model depends on several factors including death rates environmental factors. But a few areas like optimisation of malaria curing cost, role of awareness and follow-up cases, need to be researched. One of these is the coefficient of human population dependent on density. Mathematical models of the transmission of infectious agents in human communities have been studied by Anderson and May (1991). Modelling of malaria epidemic has been carried out since 1911 with Ross's model by Bailey (1957). Ghosh et al. (2014) developed a mathematical model for malaria transmission among birds by changing vector behaviour. Fatmawati and Tasman (2015) studied a mathematical model for malaria transmission by considering the resistance of malaria parasites to the anti-malarial drugs and also incorporated mass treatment and insecticides

\footnotetext{
*Corresponding author (shoketali87@gmail.com; (10) https://orcid.org/0000-0003-0383-7773)
} 
as control strategies. A new age-structured deterministic model for assessing the impact of anti-malaria drugs on the transmission dynamics of malaria was designed and qualitatively analysed by Forouzannia and Gumel (2015). Wang et al. (2016) a stage structured mosquito model was developed to investigate the cause of the large abundance of mosquitoes in Guangdong province of China in 2014 and its implications for outbreaks of the disease. Cai et al. (2017) developed a malaria model with an asymptomatic class in human population and exposed classes in both human and vector populations. The model assumes that asymptomatic individuals can get re-infected and move to the symptomatic class. Some of the developed models include density dependent death rates, environmental and other factors. However, in these studies the effect of transmission coefficient for humans dependent on the density of mosquitoes has not been considered. In this study, authors developed a susceptible, exposed, infectious and recovered (SEIR) model for humans and a susceptible, exposed and infectious (SEI) model for mosquitoes and concluded that only human immune system can fight with such a disease.

Many mathematical models have been developed earlier by many researchers on transmission dynamics of malaria disease, however the exposed class has not been taken into consideration. The exposed class is a very significant compartment, which is a stage where the disease has been transmitted to the human, but its symptoms have not yet appeared. We have incorporated an exposed class of humans in our model and analysed its effects on dynamics. Our model also considered an exposed class for mosquito population which is also an important component of the study. This has not been studied earlier studied (Singh et al. 2005; Koella, 1991; cf. Hazarika \& Bhattacharjee, 2011).

\section{MODEL DESCRIPTION}

In this model we assumed that there are two types of populations: (i) human population and (ii) vector (mosquito) population. The total number of individuals in the human population is denoted by $\mathrm{N}_{h}$ which is further subdivided into the following four subclasses:

(i) Susceptible human $\left(\mathrm{S}_{\mathrm{h}}\right)$

(ii) Exposed human $\left(\mathrm{E}_{\mathrm{h}}\right)$

(iii) Infected human $\left(\mathrm{I}_{\mathrm{h}}\right)$

(iv) Recovered human $\left(\mathrm{R}_{\mathrm{h}}\right)$.

It is assumed that people enter the susceptible class. When an infected mosquito bites the susceptible human, the susceptible human firstly may be exposed. After that there may be more infected cases with some different rates. After some time, the infected human recovers and moves to the recovered class. Once infected population is recovered, they return to the susceptible class. In this case total population is given as $\mathrm{N}_{h}=\mathrm{S}_{h}+\mathrm{E}_{\mathrm{h}}+\mathrm{I}_{\mathrm{h}}+\mathrm{R}_{\mathrm{h}}$.

Similarly, the total vector population is denoted by $\mathrm{N}_{\mathrm{v}}$ and subdivided into three classes viz. (i) susceptible mosquito $\left(\mathrm{S}_{\mathrm{v}}\right)$, (ii) exposed mosquito $\left(\mathrm{E}_{\mathrm{v}}\right)$ and (iii) infected mosquito $\left(\mathrm{I}_{\mathrm{v}}\right)$. Susceptible mosquitoes are recruited at a rate $\Lambda_{2}$. The mosquito firstly moves to the exposed class and after some time there will be more infected moves from exposed class to the infected class. In this case $\mathrm{N}_{\mathrm{v}}=\mathrm{S}_{\mathrm{v}}+\mathrm{E}_{\mathrm{v}}+\mathrm{I}_{\mathrm{v}}$. The transmission diagram of malaria is depicted in Figure (1).

For the mathematical formulation of the model, the following notations are used: $\Lambda_{1}$ : recruitment rate i.e. natural birth rate of human population; $\Lambda_{2}$ : recruitment rate of mosquito population which also includes newly born vector; $A$ : immigration rate of human population; $\beta_{1}$ : transmission probability rate from susceptible human to exposed vector (mosquito); $\beta_{2}$ : transmission probability rate from susceptible human to infected vector (mosquito); $\beta_{3}$ : transmission probability rate from susceptible vector to exposed human population; $\beta_{4}$ : transmission probability rate from susceptible vector to infected human population; $\gamma_{h}:$ progression rate from exposed class $E_{h}$ to infected class $I_{h} ; \gamma_{v}$ : progression rate from exposed class $E_{v}$ to infected class $I_{v} ; \delta$ : recovered humans rate to lose their immunity; $\xi$ : recovery rate for human population; $d_{1}\left(d_{2}\right)$ : natural death rate of human population (mosquito population).

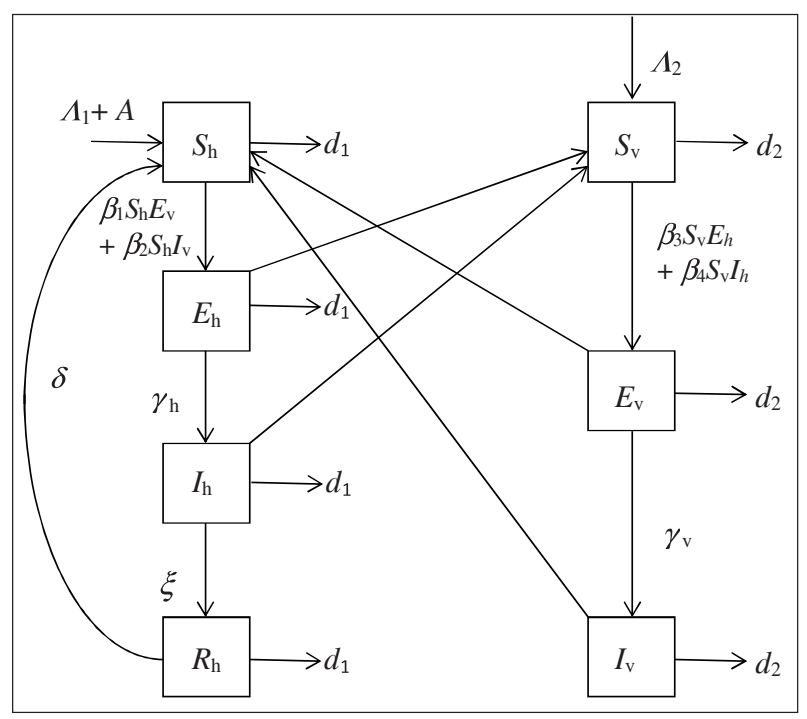

Figure 1: Model flow diagram for malaria disease transmission 
The following two cases have been taken into account to formulate the mathematical model. We will analyse the model under two different cases mentioned below:

Case 1: The transmission probability, i.e. the rate $\beta_{1}$ at which infected mosquito bites the susceptible human is assumed to be constant

Case 2: Transmission probability $\beta_{1}$ is a function of density of the mosquito population. It takes the form $\beta_{1}=b_{0}+b_{1} N_{v} ; b_{0}$ and is a positive constant.

\section{Governing equations}

The model is governed by the following system of differential equations

$$
\begin{aligned}
& \frac{d S_{h}}{d t}=\left(\Lambda_{1}+A\right)+\delta R_{h}-\left(\beta_{1} E_{v}+\beta_{2} I_{v}\right) S_{h}-d_{1} S_{h} \\
& \frac{d E_{h}}{d t}=\left(\beta_{1} E_{v}+\beta_{2} I_{v}\right) S_{h}-\left(\gamma_{h}+d_{1}\right) E_{h} \\
& \frac{d I_{h}}{d t}=\gamma_{h} E_{h}-\left(\xi+d_{1}\right) I_{h} \\
& \frac{d R_{h}}{d t}=\xi I_{h}-\left(\delta+d_{1}\right) R_{h} \\
& \frac{d N_{h}}{d t}=\Lambda_{1}+A-d_{1} N_{h} \\
& \frac{d S_{v}}{d t}=\Lambda_{2}-\left(\beta_{3} E_{h}+\beta_{4} I_{h}\right) S_{v}-d_{2} S_{v} \\
& \frac{d E_{v}}{d t}=\left(\beta_{3} E_{h}+\beta_{4} I_{h}\right) S_{v}-\left(\gamma_{v}+d_{2}\right) E_{v} \\
& \frac{d I_{v}}{d t}=\gamma_{v} E_{v}-d_{2} I_{v} \\
& \frac{d N_{v}}{d t}=\Lambda_{2}-d_{2} N_{v}
\end{aligned}
$$

\section{Mathematical Analysis}

The developed mathematical model can be discussed in the following two cases:

Case 1: When $\beta_{1}=\beta_{0} ; \beta_{0}$ is a constant

Since $S_{h}+E_{h}+I_{h}+R_{h}=N_{h}$ and $S_{v}+E_{v}+I_{v}=N_{v}$ then the system of equation (1) can be rewritten in the following form

$$
\frac{d E_{h}}{d t}=\left(\beta_{0} E_{v}+\beta_{2} I_{v}\right)\left(N_{h}-\left(E_{h}+I_{h}+R_{h}\right)\right)-\left(\gamma_{h}+d_{1}\right) E_{h}
$$

$\frac{d I_{h}}{d t}=\gamma_{h} E_{h}-\left(\xi+d_{1}\right) I_{h}$

$\frac{d R_{h}}{d t}=\xi I_{h}-\left(\delta+d_{1}\right) R_{h}$

$\frac{d N_{h}}{d t}=\Lambda_{1}+A-d_{1} N_{h}$

$\frac{d E_{v}}{d t}=\left(\beta_{3} E_{h}+\beta_{4} I_{h}\right)\left(N_{v}-\left(E_{v}+I_{v}\right)\right)-\left(\gamma_{v}+d_{2}\right) E_{v}$

$\frac{d I_{v}}{d t}=\gamma_{v} E_{v}-d_{2} I_{v}$

$\frac{d N_{v}}{d t}=\Lambda_{2}-d_{2} N_{v}$

The region of attraction of the above system is

$\Phi_{1}=\left\{\left(E_{h}, I_{h}, R_{h}, N_{h}, E_{v}, I_{v}, N_{v}\right): 0 \leq E_{h}+I_{h}+R_{h}\right.$

$\left.\leq N_{h} \leq \bar{N}_{h}, 0 \leq E_{v}+I_{v} \leq N_{v} \leq \bar{N}_{v}\right\}$

where $\bar{N}_{h}=\lim _{t \rightarrow \infty} \sup N_{h}=\frac{\Lambda_{1}+A}{d_{1}}$ and

$\bar{N}_{v}=\lim _{t \rightarrow \infty} \sup N_{v}=\frac{\Lambda_{2}}{d_{2}}$.

\section{Equilibrium points and stability analysis}

Since we know that at equilibrium state all the derivatives vanish

i.e. $\frac{d E_{h}}{d t}=\frac{d I_{h}}{d t}=\frac{d R_{h}}{d t}=\frac{d N_{h}}{d t}=\frac{d E_{v}}{d t}=\frac{d I_{v}}{d t}=\frac{d N_{v}}{d t}=0$,

then the system of equations (2) becomes

$$
\begin{aligned}
& \left(\beta_{0} E_{v}+\beta_{2} I_{v}\right)\left(N_{h}-\left(E_{h}+I_{h}+R_{h}\right)\right)-\left(\gamma_{h}+d_{1}\right) E_{h}=0 \\
& \gamma_{h} E_{h}-\left(\xi+d_{1}\right) I_{h}=0 \\
& \xi I_{h}-\left(\delta+d_{1}\right) R_{h}=0 \\
& \Lambda_{1}+A-d_{1} N_{h}=0 \\
& \left(\beta_{3} E_{h}+\beta_{4} I_{h}\right)\left(N_{v}-\left(E_{v}+I_{v}\right)\right)-\left(\gamma_{v}+d_{2}\right) E_{v}=0 \\
& \gamma_{v} E_{v}-d_{2} I_{v}=0 \\
& \Lambda_{2}-d_{2} N_{v}=0
\end{aligned}
$$


The following are three physically as well as biologically relevant equilibrium points:

(i) Disease free equilibrium for only the human population is $E_{1}=\left(0,0,0, \frac{\Lambda_{1}+A}{d_{1}}, 0,0,0\right)$

(ii) Disease free equilibrium for both human and mosquito populations $E_{2}=\left(0,0,0, \frac{\Lambda_{1}+A}{d_{1}}, 0,0, \frac{\Lambda_{2}}{d_{2}}\right)$

(iii) Endemic equilibrium point is $E_{3}=\left(\widetilde{E}_{h}, \widetilde{I}_{h}, \widetilde{R}_{h}\right.$, $\left.\widetilde{N}_{h}, \widetilde{E}_{v}, \widetilde{I}_{v}, \widetilde{N}_{v}\right)$, where

$$
\widetilde{E}_{h}=\frac{\left(\beta_{0}+\frac{\beta_{2} \gamma_{v}}{d_{2}}\right) E_{v} \bar{N}_{h}}{\left(\beta_{0}+\frac{\beta_{2} \gamma_{v}}{d_{2}}\right)\left(1+\frac{\gamma_{h}}{d_{1}+\xi}+\frac{\gamma_{h} \xi}{\left(d_{1}+\xi\right)\left(d_{1}+\delta\right)}\right) \widetilde{E}_{v}+\left(d_{1}+\gamma_{h}\right)},
$$

$$
\tilde{I}_{h}=\frac{\gamma_{h} \widetilde{E}_{h}}{d_{1}+\xi}, \widetilde{R}_{h}=\frac{\xi \gamma_{h} \widetilde{E}_{h}}{\left(d_{1}+\xi\right)\left(d_{1}+\delta\right)}, \tilde{N}_{h}=\frac{\Lambda_{1}+A}{d_{1}}
$$

$$
\tilde{E}_{v}=\frac{\left(\beta_{3}+\frac{\beta_{4} \gamma_{h}}{d_{1}+\xi}\right) \widetilde{E}_{h} \bar{N}_{v}}{\left(\beta_{3}+\frac{\beta_{4} \gamma_{h}}{d_{1}+\xi}\right)\left(1+\frac{\gamma_{v}}{d_{1}+\xi}\right) \tilde{E}_{h}+\left(d_{2}+\gamma_{v}\right)},
$$

$\tilde{I}_{v}=\frac{\gamma_{v} \widetilde{E}_{v}}{d_{2}}, \tilde{N}_{v}=\frac{\Lambda_{2}}{d_{2}}$.

Theorem 1: The equilibrium point $E_{1}$ is stable if all latent roots are negative. The equilibrium $E_{2}$ is stable if $p_{i}(i=1,2,4)>0$ and $p_{3}\left(p_{1} p_{2}-p_{3}\right)>p_{1}^{2} p_{4}$, and otherwise unstable. The equilibrium $E_{3}$ is stable if $q_{i}(i=1,3,4,5)>0$ and $\left(q_{1} q_{4}-q_{5}\right)\left(q_{1} q_{2} q_{3}-q_{1}^{2}-q_{3}^{2}-q_{1}^{2} q_{4}\right)$ $>q_{5}\left(q_{1} q_{2}-q_{3}\right)^{2}+q_{1} q_{5}^{2}$.

Proof: The general variational matrix J corresponding to the system (2) is

$$
J=\left(\begin{array}{cccc}
-\left(\beta_{0} E_{v}+\beta_{2} I_{v}\right)-\left(\gamma_{h}+d_{1}\right) & -\left(\beta_{0} E_{v}+\beta_{2} I_{v}\right) & -\left(\beta_{0} E_{v}+\beta_{2} I_{v}\right) & \left(\beta_{0} E_{v}+\beta_{2} I_{v}\right) \\
\gamma_{h} & -\left(d_{1}+\xi\right) & 0 & 0 \\
0 & \xi & -\left(d_{1}+\delta\right) & 0 \\
0 & 0 & 0 & -d_{1} \\
\beta_{3}\left(N_{v}-\left(E_{v}+I_{v}\right)\right) & \beta_{4}\left(N_{v}-\left(E_{v}+I_{v}\right)\right) & 0 & 0 \\
0 & 0 & 0 & 0 \\
0 & 0 & 0 & 0 \\
\beta_{0}\left(N_{h}-\left(E_{h}+I_{h}+R_{h}\right)\right) & \beta_{2}\left(N_{h}-\left(E_{h}+I_{h}+R_{h}\right)\right) & 0 \\
0 & 0 & 0 \\
0 & 0 & 0 \\
0 & 0 & 0 \\
-\left(\beta_{3} E_{h}+\beta_{4} I_{h}\right)-\left(\gamma_{v}+d_{2}\right) & -\left(\beta_{3} E_{h}+\beta_{4} I_{h}\right) & \left(\beta_{3} E_{h}+\beta_{4} I_{h}\right) \\
\gamma_{v} & -d_{2} & 0 \\
0 & 0 & -d_{2}
\end{array}\right)
$$

At the equilibrium point $E_{1}=\left(0,0,0, \frac{\Lambda_{1}+A}{d_{1}}, 0,0,0\right)$, the variational matrix $J_{0}$ is given by

$$
J_{0}=\left(\begin{array}{ccccccc}
-\left(\gamma_{h}+d_{1}\right) & 0 & 0 & 0 & \beta_{0}\left(\frac{\Lambda_{1}+A}{d_{1}}\right) & \beta_{2}\left(\frac{\Lambda_{1}+A}{d_{1}}\right) & 0 \\
\gamma_{h} & -\left(d_{1}+\xi\right) & 0 & 0 & 0 & 0 & 0 \\
0 & \xi & -\left(d_{1}+\delta\right) & 0 & 0 & 0 & 0 \\
0 & 0 & 0 & -d_{1} & 0 & 0 & 0 \\
0 & 0 & 0 & 0 & -\left(\gamma_{v}+d_{2}\right) & 0 & 0 \\
0 & 0 & 0 & 0 & \gamma_{v} & -d_{2} & 0 \\
0 & 0 & 0 & 0 & 0 & 0 & -d_{2}
\end{array}\right)
$$


The characteristics polynomial of the Jacobian matrix is given by $|J-\lambda I|=0$

$$
\begin{aligned}
\Rightarrow & \left(d_{1}+\lambda\right)\left(d_{2}+\lambda\right)^{2}\left(d_{1}+\delta+\lambda\right)\left(d_{1}+\xi+\lambda\right)\left(d_{1}+\gamma_{h}+\lambda\right) \\
& \left(d_{1}+\gamma_{v}+\lambda\right)=0
\end{aligned}
$$

Hence all these roots are negative. Thus the equilibrium point $E_{1}$ is stable.

At the equilibrium point $E_{2}=\left(0,0,0, \frac{\Lambda_{1}+A}{d_{1}}, 0,0, \frac{\Lambda_{2}}{d_{2}}\right)$, the variational matrix $J_{1}$ is given by

$$
J_{1}=\left(\begin{array}{ccccccc}
-\left(\gamma_{h}+d_{1}\right) & 0 & 0 & 0 & \beta_{0}\left(\frac{\Lambda_{1}+A}{d_{1}}\right) & \beta_{2}\left(\frac{\Lambda_{1}+A}{d_{1}}\right) & 0 \\
\gamma_{h} & -\left(d_{1}+\xi\right) & 0 & 0 & 0 & 0 & 0 \\
0 & \xi & -\left(d_{1}+\delta\right) & 0 & 0 & 0 & 0 \\
0 & 0 & 0 & -d_{1} & 0 & 0 & 0 \\
\beta_{3} \frac{\Lambda_{2}}{d_{2}} & \beta_{4} \frac{\Lambda_{2}}{d_{2}} & 0 & 0 & -\left(\gamma_{v}+d_{2}\right) & 0 & 0 \\
0 & 0 & 0 & 0 & \gamma_{v} & -d_{2} & 0 \\
0 & 0 & 0 & 0 & 0 & 0 & -d_{2}
\end{array}\right)
$$

The characteristics polynomial of the Jacobian matrix is given by

$$
\begin{aligned}
& |J-\lambda I|=0 \\
& \Rightarrow\left(d_{1}+\lambda\right)\left(d_{2}+\lambda\right)\left(a_{1}+\lambda\right)\left\{\lambda^{4}+\lambda^{3} p_{1}+\lambda^{2} p_{2}+\lambda p_{3}+p_{4}\right\}=0
\end{aligned}
$$

where

$$
\begin{aligned}
p_{1}= & a_{2}+a_{3}+a_{4}+d_{2}, \\
p_{2}= & a_{2} a_{3}+a_{2} a_{4}+a_{3} a_{4}+\left(a_{2}+a_{3}+a_{4}\right) d_{2}-\beta_{1} \beta_{3} N_{h} N_{v}, \\
p_{3}= & a_{2} a_{3} a_{4}+\left(a_{2} a_{3}+a_{2} a_{4}+a_{3} a_{4}\right) d_{2}-\beta_{1} \beta_{4} \gamma_{h} N_{h} N_{v}- \\
& \beta_{1} \beta_{3}\left(a_{2}+d_{2}\right) N_{h} N_{v}-\beta_{1} \beta_{3} \gamma_{v} N_{h} N_{v},
\end{aligned}
$$

$p_{4}=a_{2} a_{3} a_{4} d_{2}+\left(a_{2} a_{3}+a_{2} a_{4}+a_{3} a_{4}\right) d_{2}-\left(\beta_{1} \beta_{2} \gamma_{h} d_{2}+\beta_{2} \beta_{4} \gamma_{h} \gamma_{v}\right.$

$$
\left.+\beta_{1} \beta_{3} a_{2} d_{2}+\beta_{2} \beta_{3} \gamma_{v} a_{2}\right) N_{h} N_{v},
$$

and

$$
a_{1}=\left(d_{1}+\delta\right), a_{2}=\left(d_{1}+\xi\right), a_{3}=\left(d_{1}+\gamma_{h}\right), a_{4}=\left(d_{2}+\gamma_{v}\right)
$$

The three roots of the polynomial given by equation (8) are negative and the coefficient of a biquadratic equation give all roots with negative real part. Hence Routh-Hurwitz conditions are: $\quad p_{i}(i=1,2,4)>0$ and $p_{3}\left(p_{1} p_{2}-p_{3}\right)>p_{1}^{2} p_{4}$. For these conditions the equilibrium points $E_{2}$ is locally asymptotically stable.

At the equilibrium point $E_{3}=\left(\widetilde{E}_{h}, \widetilde{I}_{h}, \widetilde{R}_{h}, \widetilde{N}_{h}, \widetilde{E}_{v}, \widetilde{I}_{v}\right.$, $\tilde{N}_{v}$ ), the variational matrix $J_{2}$ is given by

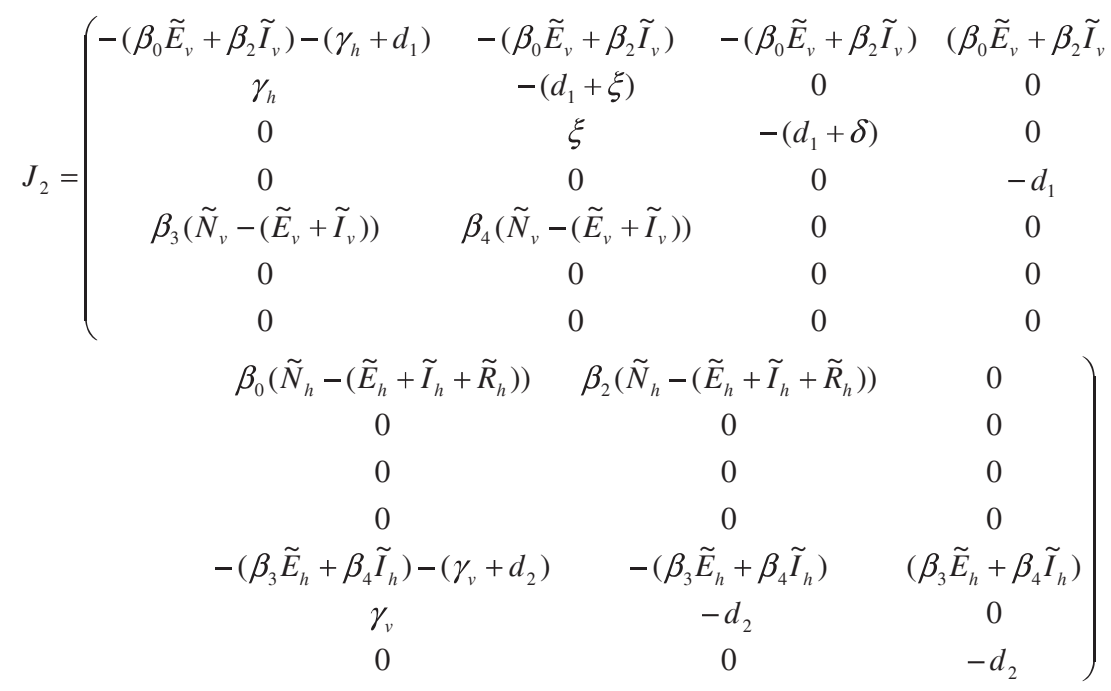


The characteristics polynomial of the Jacobian matrix is given by

$$
\begin{aligned}
& |J-\lambda I|=0 \\
& \Rightarrow\left(d_{1}+\lambda\right)\left(d_{2}+\lambda\right)\left\{\lambda^{5}+\lambda^{4} q_{1}+\lambda^{3} q_{2}+\lambda^{2} q_{3}+\lambda q_{4}+q_{5}\right\}=0
\end{aligned}
$$

where

$$
\begin{aligned}
q_{1}= & a_{1}+a_{2}+a_{3}+a_{4}+a_{5}+a_{6}+d_{2}, \\
q_{2}= & \left(\left(a_{4}+a_{6}\right) d_{2}+a_{6} \gamma_{v}\right)+\left(a_{1}+a_{2}+a_{3}+a_{5}\right)\left(a_{4}+a_{6}+d_{2}\right) \\
& +\left(a_{1} a_{2}+a_{1} a_{3}+a_{1} a_{5}+a_{2} a_{3}+a_{2} a_{5}\right)+a_{7} a_{8} \beta_{1} \beta_{3}-\gamma_{h} a_{5}, \\
q_{3}= & \left(a_{1}+a_{2}+a_{3}+a_{5}\right)\left(\left(a_{4}+a_{6}\right) d_{2}+a_{6} \gamma_{v}\right)+\left(a_{1} a_{2} a_{3}+\right. \\
& \left.a_{1} a_{2} a_{5}\right)+\left(a_{1} a_{2}+a_{1} a_{3}+a_{1} a_{5}+a_{2} a_{3}+a_{2} a_{5}\right)\left(a_{4}+a_{6}+d_{2}\right) \\
& +a_{7} a_{8} \beta_{3}\left(\beta_{1} d_{2}+a_{1} \beta_{1}+a_{2} \beta_{1}+\beta_{2} \gamma_{v}\right)+a_{7} a_{8} \beta_{1} \beta_{4}+\gamma_{h} \xi_{5} \\
& -\gamma_{h} a_{5}\left(a_{1}+a_{4}+a_{6}+d_{2}\right), \\
q_{4}= & \left(a_{1} a_{2}+a_{1} a_{3}+a_{1} a_{5}+a_{2} a_{3}+a_{2} a_{5}\right)\left(\left(a_{4}+a_{6}\right) d_{2}+a_{6} \gamma_{v}\right) \\
& +\left(a_{1} a_{2} a_{3}+a_{1} a_{2} a_{5}\right)\left(a_{4}+a_{6}+d_{2}\right)+\gamma_{h} \xi a_{5}\left(a_{4}+a_{6}+d_{2}\right) \\
& +a_{7} a_{8} \beta_{4}\left(\beta_{1} d_{2}+a_{1} \beta_{1}+\beta_{2} \gamma_{v}\right)+a_{7} a_{8} \beta_{3}\left(a_{1} \beta_{1} d_{2}+a_{2} \beta_{1} d_{2}\right. \\
& \left.+a_{1} a_{2} \beta_{1}+a_{1} \beta_{2} \gamma_{v}+a_{2} \beta_{2} \gamma_{v}\right)-\gamma_{h} a_{5}\left(a_{1}\left(a_{4}+a_{6}+d_{2}\right)\right. \\
& +\left(a_{6} \gamma_{v}\right)+a_{7} a_{8} \beta_{4}\left(\beta_{1} a_{1} d_{2}+\beta_{2} \gamma_{v}\right)-\gamma_{h} a_{5}\left(\left(a_{4}+a_{6}\right) a_{2}+a_{2}+a_{7} a_{8} \beta_{3}\left(a_{1} a_{6} \gamma_{2} \beta_{1} d_{2}\right.\right. \\
q_{5}= & \left(a_{1} a_{2} a_{3}+a_{1} a_{2} a_{5}\right)\left(\left(a_{4}+a_{6}\right) d_{2}+a_{6} \gamma_{v}\right)+\gamma_{h} \xi a_{5}\left(\left(a_{4}+a_{6}\right) d_{2}\right. \\
& \left.+a_{1}\right)
\end{aligned}
$$

and

$$
\begin{aligned}
& a_{1}=\left(d_{1}+\delta\right), a_{2}=\left(d_{1}+\xi\right), a_{3}=\left(d_{1}+\gamma_{h}\right), a_{4}=\left(d_{2}+\gamma_{v}\right), \\
& a_{5}=\left(\beta_{0} E_{v}+\beta_{2} I_{v}\right), a_{6}=\left(\beta_{3} E_{h}+\beta_{4} I_{h}\right) \\
& a_{7}=\left(N_{h}-\left(E_{h}+I_{h}+R_{h}\right)\right), a_{8}=\left(N_{v}-\left(E_{v}+I_{v}\right)\right) .
\end{aligned}
$$

Two roots are negative and the coefficients of the above equation give all roots with negative real part. Hence Routh-Hurwitz conditions are: $\quad q_{i}(i=1,3,4,5)>0$ and $\quad\left(q_{1} q_{4}-q_{5}\right)$ $\left(q_{1} q_{2} q_{3}-q_{1}^{2}-q_{3}^{2}-q_{1}^{2} q_{4}\right)>q_{5}\left(q_{1} q_{2}-q_{3}\right)^{2}+q_{1} q_{5}^{2}$. For these conditions the equilibrium points $E_{3}$ is locally asymptotically stable.

Case 2: When $\beta_{1}=b_{0}+b_{1} N_{v} ; b_{0}$ and $b_{1}$ is a constant

Since $S_{h}+E_{h}+I_{h}+R_{h}=N_{h}$ and $S_{v}+E_{v}+I_{v}=N_{v}$ the system of equation (1) can be rewritten in the following form

$$
\begin{aligned}
& \frac{d E_{h}}{d t}=\left(b_{0}+b_{1} N_{v}\right)\left(N_{h}-\left(E_{h}+I_{h}+R_{h}\right)\right) E_{v} \\
& +\beta_{2} I_{v}\left(N_{h}-\left(E_{h}+I_{h}+R_{h}\right)\right)-\left(\gamma_{h}+d_{1}\right) E_{h} \\
& \frac{d I_{h}}{d t}=\gamma_{h} E_{h}-\left(\xi+d_{1}\right) I_{h} \\
& \frac{d R_{h}}{d t}=\xi I_{h}-\left(\delta+d_{1}\right) R_{h} \\
& \frac{d N_{h}}{d t}=\Lambda_{1}+A-d_{1} N_{h} \\
& \frac{d E_{v}}{d t}=\left(\beta_{3} E_{h}+\beta_{4} I_{h}\right)\left(N_{v}-\left(E_{v}+I_{v}\right)\right)-\left(\gamma_{v}+d_{2}\right) E_{v} \\
& \frac{d I_{v}}{d t}=\gamma_{v} E_{v}-d_{2} I_{v} \\
& \frac{d N_{v}}{d t}=\Lambda_{2}-d_{2} N_{v}
\end{aligned}
$$

The region of attraction of the system given by equation (10) is

$$
\begin{gathered}
\Phi_{2}=\left\{\left(E_{h}, I_{h}, R_{h}, N_{h}, E_{v}, I_{v}, N_{v}\right): 0 \leq E_{h}+I_{h}+R_{h}\right. \\
\left.\leq N_{h} \leq \hat{N}_{h}, 0 \leq E_{v}+I_{v} \leq N_{v} \leq \hat{N}_{v}\right\}
\end{gathered}
$$

where $\hat{N}_{h}=\lim _{t \rightarrow \infty} \sup N_{h}=\frac{\Lambda_{1}+A}{d_{1}}$ and

$\widehat{N}_{v}=\lim _{t \rightarrow \infty} \sup N_{v}=\frac{\Lambda_{2}}{d_{2}}$.

\section{Equilibrium points and stability analysis}

Since we know that at equilibrium state all the derivatives vanish 
i.e. $\frac{d E_{h}}{d t}=\frac{d I_{h}}{d t}=\frac{d R_{h}}{d t}=\frac{d N_{h}}{d t}=\frac{d E_{v}}{d t}=\frac{d I_{v}}{d t}=\frac{d N_{v}}{d t}=0$,

then the system of equations (10) becomes

$\left(b_{0}+b_{1} N_{v}\right)\left(N_{h}-\left(E_{h}+I_{h}+R_{h}\right)\right) E_{v}+\beta_{2} I_{v}\left(N_{h}-\left(E_{h}+\right.\right.$

$\left.\left.I_{h}+R_{h}\right)\right)-\left(\gamma_{h}+d_{1}\right) E_{h}=0$

$\gamma_{h} E_{h}-\left(\xi+d_{1}\right) I_{h}=0$

$\xi I_{h}-\left(\delta+d_{1}\right) R_{h}=0$

$\Lambda_{1}+A-d_{1} N_{h}=0$

$\left(\beta_{3} E_{h}+\beta_{4} I_{h}\right)\left(N_{v}-\left(E_{v}+I_{v}\right)\right)-\left(\gamma_{v}+d_{2}\right) E_{v}=0$

$\gamma_{v} E_{v}-d_{2} I_{v}=0$
$\Lambda_{2}-d_{2} N_{v}=0$

The following are three physically as well as biologically relevant equilibrium points:

(i) When the exposed human population is infected then the disease free equilibrium only for human population is $P_{1}=\left(E_{h}^{\prime}, 0,0, \frac{\Lambda_{1}+A}{d_{1}}, 0,0,0\right)$

(ii) When the exposed human population is infected only then the disease free equilibrium for both human and mosquito populations is $P_{2}=\left(E_{h}^{\prime}, 0,0, \frac{\Lambda_{1}+A}{d_{1}}, 0,0, \frac{\Lambda_{2}}{d_{2}}\right)$

(iii) Endemic equilibrium point is $P_{3}=\left(\widetilde{E}_{h}, \tilde{I}_{h}, \widetilde{R}_{h}, \tilde{N}_{h}\right.$, $\left.\tilde{E}_{v}, \tilde{I}_{v}, \tilde{N}_{v}\right)$, where

$E_{h}^{\prime}=\frac{\left(b_{0}+b_{1} \frac{\Lambda_{2}}{d_{2}}+\frac{\beta_{2} \gamma_{v}}{d_{2}}\right) \tilde{E}_{v} \frac{\Lambda_{1}+A}{d_{1}}}{\left(b_{0}+b_{1} \frac{\Lambda_{2}}{d_{2}}+\frac{\beta_{2} \gamma_{v}}{d_{2}}\right)\left(1+\frac{\gamma_{h}}{d_{1}+\xi}+\frac{\gamma_{h} \xi}{\left(d_{1}+\xi\right)\left(d_{1}+\delta\right)}\right) \tilde{E}_{v}+\left(d_{1}+\gamma_{h}\right)}$,

$\tilde{E}_{h}=\frac{\left(\left(b_{0}+b_{1} N_{v}\right)+\frac{\beta_{2} \gamma_{v}}{d_{2}}\right) \tilde{E}_{v} \hat{N}_{h}}{\left(\left(b_{0}+b_{1} N_{v}\right)+\frac{\beta_{2} \gamma_{v}}{d_{2}}\right)\left(1+\frac{\gamma_{h}}{d_{1}+\xi}+\frac{\gamma_{h} \xi}{\left(d_{1}+\xi\right)\left(d_{1}+\delta\right)}\right) \tilde{E}_{v}+\left(d_{1}+\gamma_{h}\right)}$,

$\tilde{I}_{h}=\frac{\gamma_{h} \tilde{E}_{h}}{d_{1}+\xi}, \tilde{R}_{h}=\frac{\xi \gamma_{h} \tilde{E}_{h}}{\left(d_{1}+\xi\right)\left(d_{1}+\delta\right)}, \tilde{N}_{h}=\frac{\Lambda_{1}+A}{d_{1}}, \quad \tilde{I}_{v}=\frac{\gamma_{v} \tilde{E}_{v}}{d_{2}}, \tilde{N}_{v}=\frac{\Lambda_{2}}{d_{2}}$

...(14) Theorem 2: The equilibrium point $P_{1}$ is stable if all $\tilde{E}_{v}=\frac{\left(\beta_{3}+\frac{\beta_{4} \gamma_{h}}{d_{1}+\xi}\right) \widetilde{E}_{h} \widehat{N}_{v}}{\left(\beta_{3}+\frac{\beta_{4} \gamma_{h}}{d_{1}+\xi}\right)\left(1+\frac{\gamma_{v}}{d_{1}+\xi}\right) \tilde{E}_{h}+\left(d_{2}+\gamma_{v}\right)}$, latent roots are negative. The equilibrium $P_{2}$ is stable if $m_{i}(i=1,2,4)>0$ and $m_{3}\left(m_{1} m_{2}-m_{3}\right)>m_{1}^{2} m_{4}$, otherwise unstable and the equilibrium $P_{3}$ is stable if

$s_{i}(i=1,3,4,5)>0$ and $\left(s_{1} s_{4}-s_{5}\right)\left(s_{1} s_{2} s_{3}-s_{1}^{2}-s_{3}^{2}-\right.$ $\left.-s_{1}^{2} s_{4}\right)>s_{5}\left(s_{1} s_{2}-s_{3}\right)^{2}+s_{1} s_{5}^{2}$. 
Proof: The general variational matrix M corresponding to the system (10) is:

$$
M=\left(\begin{array}{ccc}
-\left(b_{0}+b_{1} N_{v}\right) E_{v}-\beta_{2} I_{v}-\left(\gamma_{h}+d_{1}\right) & -\left(b_{0}+b_{1} N_{v}\right) E_{v}-\beta_{2} I_{v} & -\left(b_{0}+b_{1} N_{v}\right) E_{v}-\beta_{2} I_{v} \\
\gamma_{h} & -\left(d_{1}+\xi\right) & 0 \\
0 & \xi & -\left(d_{1}+\delta\right) \\
0 & 0 & 0 \\
\beta_{3}\left(N_{v}-\left(E_{v}+I_{v}\right)\right) & \beta_{4}\left(N_{v}-\left(E_{v}+I_{v}\right)\right) & 0 \\
0 & 0 & 0 \\
0 & 0 & 0
\end{array}\right.
$$

$$
\left.\begin{array}{cccc}
\left(b_{0}+b_{1} N_{v}\right) E_{v}+\beta_{2} I_{v} & \left(b_{0}+b_{1} N_{v}\right)\left(N_{h}-\left(E_{h}+I_{h}+R_{h}\right)\right) & \beta_{2}\left(N_{h}-\left(E_{h}+I_{h}+R_{h}\right)\right) & 0 \\
0 & 0 & 0 & 0 \\
0 & 0 & 0 & 0 \\
-d_{1} & 0 & 0 & 0 \\
0 & -\left(\beta_{3} E_{h}+\beta_{4} I_{h}\right)-\left(\gamma_{v}+d_{2}\right) & -\left(\beta_{3} E_{h}+\beta_{4} I_{h}\right) & \left(\beta_{3} E_{h}+\beta_{4} I_{h}\right) \\
0 & \gamma_{v} & -d_{2} & 0 \\
0 & 0 & 0 & -d_{2}
\end{array}\right)
$$

At the equilibrium point $P_{1}=\left(E_{h}^{\prime}, 0,0, \frac{\Lambda_{1}+A}{d_{1}}, 0,0,0\right)$, the variational matrix $M_{0}$ is given by

$$
M_{0}=\left(\begin{array}{ccccccc}
-\left(\gamma_{h}+d_{1}\right) & 0 & 0 & 0 & b_{0}\left(\frac{\Lambda_{1}+A}{d_{1}}-E_{h}^{\prime}\right) & \beta_{2}\left(\frac{\Lambda_{1}+A}{d_{1}}-E_{h}^{\prime}\right) & 0 \\
\gamma_{h} & -\left(d_{1}+\xi\right) & 0 & 0 & 0 & 0 & 0 \\
0 & \xi & -\left(d_{1}+\delta\right) & 0 & 0 & 0 & 0 \\
0 & 0 & 0 & -d_{1} & 0 & 0 & 0 \\
0 & 0 & 0 & 0 & -\left(\beta_{3} E_{h}^{\prime}+\gamma_{v}+d_{2}\right) & -\beta_{3} E_{h}^{\prime} & \beta_{3} E_{h}^{\prime} \\
0 & 0 & 0 & 0 & \gamma_{v} & -d_{2} & 0 \\
0 & 0 & 0 & 0 & 0 & 0 & -d_{2}
\end{array}\right)
$$

The characteristics polynomial of the Jacobian matrix is given by

$$
\begin{aligned}
& |J-\lambda I|=0 \\
\Rightarrow & \left(d_{1}+\lambda\right)\left(d_{2}+\lambda\right)\left(d_{1}+\delta+\lambda\right)\left(d_{1}+\xi+\lambda\right)\left(d_{1}+\gamma_{h}+\lambda\right)\left[\left(\left(\beta_{3} E_{h}^{\prime}+\gamma_{v}+d_{2}\right)+\lambda\right)\left(d_{2}+\lambda\right)+\beta_{3} E_{h}^{\prime} \gamma_{v}\right]=0 \\
\Rightarrow & \left(d_{1}+\lambda\right)\left(d_{2}+\lambda\right)\left(d_{1}+\delta+\lambda\right)\left(d_{1}+\xi+\lambda\right)\left(d_{1}+\gamma_{h}+\lambda\right)\left[\lambda^{2}+\left\{\left(\beta_{3} E_{h}^{\prime}+\gamma_{v}+d_{2}\right)+d_{2}\right\} \lambda\right. \\
& \left.+\left(\beta_{3} E_{h}^{\prime}+\gamma_{v}+d_{2}\right) d_{2}+\beta_{3} E_{h}^{\prime} \gamma_{v}\right]=0
\end{aligned}
$$

Obviously, five roots of the above equation are negative and the other two roots are obtained by the quadratic equations.
At the equilibrium point $P_{2}=\left(E_{h}^{\prime}, 0,0, \frac{\Lambda_{1}+A}{d_{1}}, 0,0, \frac{\Lambda_{2}}{d_{2}}\right)$, the variational matrix $M_{1}$ is given by 


$$
M_{1}=\left(\begin{array}{ccccccc}
-\left(\gamma_{h}+d_{1}\right) & 0 & 0 & 0 & \left(b_{0}+b_{1} \frac{\Lambda_{2}}{d_{2}}\right)\left(\frac{\Lambda_{1}+A}{d_{1}}-E_{h}^{\prime}\right) & \beta_{2}\left(\frac{\Lambda_{1}+A}{d_{1}}-E_{h}^{\prime}\right) & 0 \\
\gamma_{h} & -\left(d_{1}+\xi\right) & 0 & 0 & 0 & 0 & 0 \\
0 & \xi & -\left(d_{1}+\delta\right) & 0 & 0 & 0 & 0 \\
0 & 0 & 0 & -d_{1} & 0 & -\beta_{3} E_{h}^{\prime} & \beta_{3} E_{h}^{\prime} \\
\beta_{3} \frac{\Lambda_{2}}{d_{2}} & \beta_{4} \frac{\Lambda_{2}}{d_{2}} & 0 & 0 & -\left(\beta_{3} E_{h}^{\prime}+\gamma_{v}+d_{2}\right) & -d_{2} & 0 \\
0 & 0 & 0 & 0 & \gamma_{v} & 0 & -d_{2} \\
0 & 0 & 0 & 0 & 0 & 0
\end{array}\right)
$$

The characteristics polynomial of the Jacobian matrix is given by

$$
\begin{aligned}
& |J-\lambda I|=0 \\
& \Rightarrow\left(d_{1}+\lambda\right)\left(d_{2}+\lambda\right)\left(a_{1}+\lambda\right)\left\{\lambda^{4}+\lambda^{3} m_{1}+\lambda^{2} m_{2}\right. \\
& \left.+\lambda m_{3}+m_{4}\right\}=0
\end{aligned}
$$

where,

$$
\begin{aligned}
m_{1}= & a_{2}+a_{3}+a_{4}+d_{2}, \\
m_{2}= & a_{2} a_{3}+a_{2} a_{4}+a_{3} a_{4}+a_{4} d_{2}+a_{2} d_{2}+a_{3} d_{2} \\
& +\left(a_{2}+a_{3}+a_{4}\right) d_{2}+\gamma_{v} \beta_{3} E_{h}^{\prime} \\
& -\beta_{3} N_{v}\left(b_{0}+b_{1} N_{v}\right)\left(N_{h}-E_{h}^{\prime}\right), \\
m_{3}= & a_{2} a_{3} a_{4}+\left(a_{2} a_{3}+a_{2} a_{4}+a_{3} a_{4}\right) d_{2}+ \\
& \left(a_{2}+a_{3}\right) \beta_{3} \gamma_{v} E_{h}^{\prime}+\beta_{3} N_{v}\left(a_{2}+d_{2}\right)\left(b_{0}+b_{1} N_{v}\right) \\
& \left(N_{h}-E_{h}^{\prime}\right)-\beta_{2} \beta_{3} \gamma_{v} N_{v}\left(N_{h}-E_{h}^{\prime}\right) \\
& -\beta_{4} \gamma_{h} N_{v}\left(b_{0}+b_{1} N_{v}\right)\left(N_{h}-E_{h}^{\prime}\right),
\end{aligned}
$$

$m_{4}=a_{2} a_{3} a_{4} d_{2}+a_{2} a_{3} \beta_{3} \gamma_{v} E_{h}^{\prime}+a_{2} d_{2} \beta_{3} N_{v}$

$$
\begin{aligned}
& \left(b_{0}+b_{1} N_{v}\right)\left(N_{h}-E_{h}^{\prime}\right)+\beta_{2} \beta_{3} \gamma_{v} a_{2} N_{v} \\
& \left(N_{h}-E_{h}^{\prime}\right)-\beta_{4} \gamma_{h} d_{2} N_{v}\left(b_{0}+b_{1} N_{v}\right)\left(N_{h}-E_{h}^{\prime}\right) \\
& -\beta_{4} \gamma_{h} \gamma_{v} N_{v}\left(N_{h}-E_{h}^{\prime}\right),
\end{aligned}
$$

and

$a_{1}=\left(d_{1}+\delta\right), a_{2}=\left(d_{1}+\xi\right), a_{3}=\left(d_{1}+\gamma_{h}\right)$,

$a_{4}=\left(\beta_{3} E_{h}^{\prime}+\gamma_{v}+d_{2}\right)$.

The three roots of the polynomial given by equation (17) are negative and the coefficient of a biquadratic equation give all roots with negative real part. Hence Routh - Hurwitz conditions are: $m_{i}(i=1,2,4)>0$ and $m_{3}\left(m_{1} m_{2}-m_{3}\right)>m_{1}^{2} m_{4} . \quad$ For these conditions the equilibrium point $P_{2}$ is locally asymptotically stable.

At the equilibrium point $P_{3}=\left(\widetilde{E}_{h}, \widetilde{I}_{h}, \widetilde{R}_{h}, \widetilde{N}_{h}, \widetilde{E}_{v}\right.$, $\left.,, \widetilde{I}_{v}, \widetilde{N}_{v}\right)$ the variational matrix $M_{2}$ is given by

$M_{2}=\left(\begin{array}{ccc}-\left(b_{0}+b_{1} \tilde{N}_{v}\right) \tilde{E}_{v}-\beta_{2} \tilde{I}_{v}-\left(\gamma_{h}+d_{1}\right) & -\left(b_{0}+b_{1} \tilde{N}_{v}\right) \tilde{E}_{v}-\beta_{2} \tilde{I}_{v} & -\left(b_{0}+b_{1} \tilde{N}_{v}\right) \tilde{E}_{v}-\beta_{2} \tilde{I}_{v} \\ \gamma_{h} & -\left(d_{1}+\xi\right) & 0 \\ 0 & \xi & -\left(d_{1}+\delta\right) \\ 0 & 0 & 0 \\ \beta_{3}\left(\tilde{N}_{v}-\left(\tilde{E}_{v}+\tilde{I}_{v}\right)\right) & \beta_{4}\left(\tilde{N}_{v}-\left(\tilde{E}_{v}+\tilde{I}_{v}\right)\right) & 0 \\ 0 & 0 & 0 \\ 0 & 0 & 0\end{array}\right.$ 


$\left.\begin{array}{cccc}\left(b_{0}+b_{1} \tilde{N}_{v}\right) \widetilde{E}_{v}+\beta_{2} \tilde{I}_{v} & \left(b_{0}+b_{1} \tilde{N}_{v}\right)\left(\tilde{N}_{h}-\left(\widetilde{E}_{h}+\tilde{I}_{h}+\widetilde{R}_{h}\right)\right) & \beta_{2}\left(\tilde{N}_{h}-\left(\widetilde{E}_{h}+\tilde{I}_{h}+\widetilde{R}_{h}\right)\right) & 0 \\ 0 & 0 & 0 & 0 \\ 0 & 0 & 0 & 0 \\ -d_{1} & 0 & 0 & 0 \\ 0 & -\left(\beta_{3} \widetilde{E}_{h}+\beta_{4} \tilde{I}_{h}\right)-\left(\gamma_{v}+d_{2}\right) & -\left(\beta_{3} \widetilde{E}_{h}+\beta_{4} \tilde{I}_{h}\right) & \left(\beta_{3} \widetilde{E}_{h}+\beta_{4} \tilde{I}_{h}\right) \\ 0 & \gamma_{v} & -d_{2} & 0 \\ 0 & 0 & 0 & -d_{2}\end{array}\right)$

The characteristics polynomial of the Jacobian matrix is given by

$|J-\lambda I|=0$

$\Rightarrow\left(d_{1}+\lambda\right)\left(d_{2}+\lambda\right)\left\{\lambda^{5}+\lambda^{4} s_{1}+\lambda^{3} s_{2}+\lambda^{2} s_{3}+\lambda s_{4}+s_{5}\right\}=0$

where

$$
\begin{aligned}
s_{1}= & a_{1}+a_{2}+a_{3}+a_{4}+a_{5}+a_{6}+d_{2}, \\
s_{2}= & \left(\left(a_{4}+a_{6}\right) d_{2}+a_{6} \gamma_{v}\right)+\left(a_{1}+a_{2}+a_{3}+a_{5}\right) \\
& \left(a_{4}+a_{6}+d_{2}\right)+\left(a_{1} a_{2}+a_{1} a_{3}+a_{1} a_{5}+a_{2} a_{3}\right. \\
& \left.+a_{2} a_{5}\right)+a_{7} a_{8} a_{9} \beta_{3}-\gamma_{h} a_{5}, \\
s_{3}= & \left(a_{1}+a_{2}+a_{3}+a_{5}\right)\left(\left(a_{4}+a_{6}\right) d_{2}+a_{6} \gamma_{v}\right)+ \\
& \left(a_{1} a_{2} a_{3}+a_{1} a_{2} a_{5}\right)+\left(a_{1} a_{2}+a_{1} a_{3}+a_{1} a_{5}\right. \\
& \left.+a_{2} a_{3}+a_{2} a_{5}\right)\left(a_{4}+a_{6}+d_{2}\right)+a_{7} a_{8} \beta_{3}\left(a_{9} d_{2}\right. \\
& \left.+a_{1} a_{9}+a_{2} a_{9}+\beta_{2} \gamma_{v}\right)+a_{7} a_{8} a_{9} \beta_{4}+\gamma_{h} \xi_{5} \\
& -\gamma_{h} a_{5}\left(a_{1}+a_{4}+a_{6}+d_{2}\right), \\
s_{4}= & \left(a_{1} a_{2}+a_{1} a_{3}+a_{1} a_{5}+a_{2} a_{3}+a_{2} a_{5}\right)\left(\left(a_{4}+a_{6}\right) d_{2}\right. \\
& \left.+a_{6} \gamma_{v}\right)+\left(a_{1} a_{2} a_{3}+a_{1} a_{2} a_{5}\right)\left(a_{4}+a_{6}+d_{2}\right) \\
& +\gamma_{h} \xi a_{5}\left(a_{4}+a_{6}+d_{2}\right)+a_{7} a_{8} \beta_{4}\left(a_{9} d_{2}+a_{1} a_{9}+\right. \\
& \left.\beta_{2} \gamma_{v}\right)+a_{7} a_{8} \beta_{3}\left(a_{1} a_{9} d_{2}+a_{2} a_{9} d_{2}+a_{1} a_{2} a_{9}\right. \\
& \left.+a_{1} \beta_{2} \gamma_{v}+a_{2} \beta_{2} \gamma_{v}\right)-\gamma_{h} a_{5}\left(a_{1}\left(a_{4}+a_{6}+d_{2}\right)\right. \\
& \left.+\left(a_{4}+a_{6}\right) d_{2}+a_{6} \gamma_{v}\right) \\
s_{5}= & \left(a_{1} a_{2} a_{3}+a_{1} a_{2} a_{5}\right)\left(\left(a_{4}+a_{6}\right) d_{2}+a_{6} \gamma_{v}\right)+ \\
& \gamma_{h} \xi a_{5}\left(\left(a_{4}+a_{6}\right) d_{2}+a_{6} \gamma_{v}\right)+a_{7} a_{8} \beta_{4}\left(a_{1} a_{9} d_{2}\right. \\
& \left.+\beta_{2} a_{1} \gamma_{v}\right)+a_{7} a_{8} \beta_{3}\left(a_{1} a_{2} a_{9} d_{2}+a_{1} a_{2} \beta_{2} \gamma_{v}\right) \\
& -\gamma_{h} a_{5}\left(\left(a_{4}+a_{6}\right) a_{1} d_{2}+a_{1} a_{6} \gamma_{v}\right) \\
&
\end{aligned}
$$

and

$a_{1}=\left(d_{1}+\delta\right), a_{2}=\left(d_{1}+\xi\right), a_{3}=\left(d_{1}+\gamma_{h}\right)$,

$a_{4}=\left(d_{2}+\gamma_{v}\right), a_{5}=\left(b_{0}+b_{1} N_{v}\right) E_{v}+\beta_{2} I_{v}$, $a_{6}=\left(\beta_{3} E_{h}+\beta_{4} I_{h}\right), a_{7}=\left(N_{h}-\left(E_{h}+I_{h}+R_{h}\right)\right)$,

$a_{8}=\left(N_{v}-\left(E_{v}+I_{v}\right)\right), a_{9}=b_{0}+b_{1} N_{v}$.

Two roots are negative and the coefficients of the above equation give all roots with negative real part. Hence Routh-Hurwitz conditions are:

$s_{i}(i=1,3,4,5)>0$ and $\left(s_{1} s_{4}-s_{5}\right)\left(s_{1} s_{2} s_{3}-s_{1}^{2}-s_{3}^{2}\right.$

$\left.-s_{1}^{2} s_{4}\right)>s_{5}\left(s_{1} s_{2}-s_{3}\right)^{2}+s_{1} s_{5}^{2}$.

Under these conditions the equilibrium point $P_{3}$ is locally asymptotically stable.

The global stability of the above equilibriums can be computed by constructing the Lyapunov function.

\section{RESULTS AND DISCUSSION}

The numerical simulation is presented to validate the analytical results discussed previously. For this purpose, Runge-Kutta fourth-order method was used to perform the sensitivity analysis. The dataset for case 1 was as follows:

$\beta_{0}=0.00000029 ; \quad \beta_{2}=0.00000021 ; \quad \beta_{3}=0.00000015 ;$

$\beta_{4}=0.00000009 ; \quad d_{1}=0.00012 ; \quad d_{2}=0.0085 ;$

$\Lambda_{1}=0.00012 ; \quad \Lambda_{2}=0.0085 ; \quad \delta=0.00146 ; \quad \xi=0.0085 ;$

$\gamma_{1}=0.012 ; \gamma_{2}=0.015 ; \mathrm{A}=12$;

and initial values were:

$E_{h}=500 ; I_{h}=4181.8 ; R_{h}=209.2 ; N_{h}=20000 ; E_{v}=14000 ;$

$I_{v}=30000 ; N_{v}=963400$,

which are based on Gosh et al. (2005) and Hazarika and Bhattacharjee (2011).

Similarly, for case 2, the parameters values are taken as follows: 
$b_{0}=0.00012 ; \quad b_{1}=0.00000006 ; \quad \beta_{2}=0.00000021 ;$

$\beta_{3}=0.000024 ; \quad \beta_{4}=000000009 ; \quad d_{1}=0.00012 ;$

$d_{2}=0.0085 ; \quad \Lambda_{1}=0.00012 ; \Lambda_{2}=0.00085 ; \delta=0.000146 ;$

$\xi=0.012 ; \gamma_{1}=0.012 ; \gamma_{2}=0.015 ; \mathrm{A}=10 ;$

and initial values were:

$E_{h}=500 ; \quad I_{h}=4181.8 ; \quad R_{h}=209.2 ; \quad N_{h}=20000 ;$

$E_{v}=14000 ; I_{v}=30000 ; N_{v}=963400$.

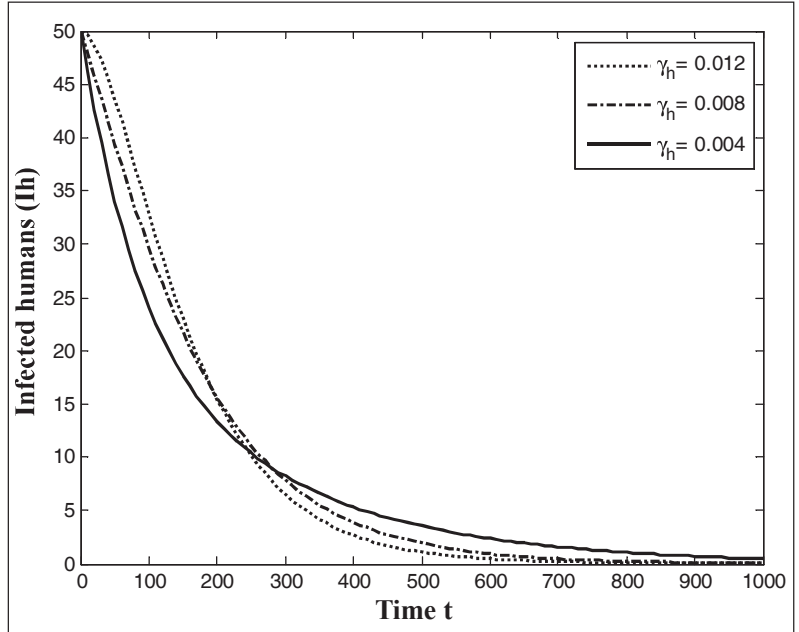

Figure 2: Variation in infective human population $I_{h}$ with time for various values of progression rate $\gamma_{h}$, when the transmission coefficient for humans is a constant

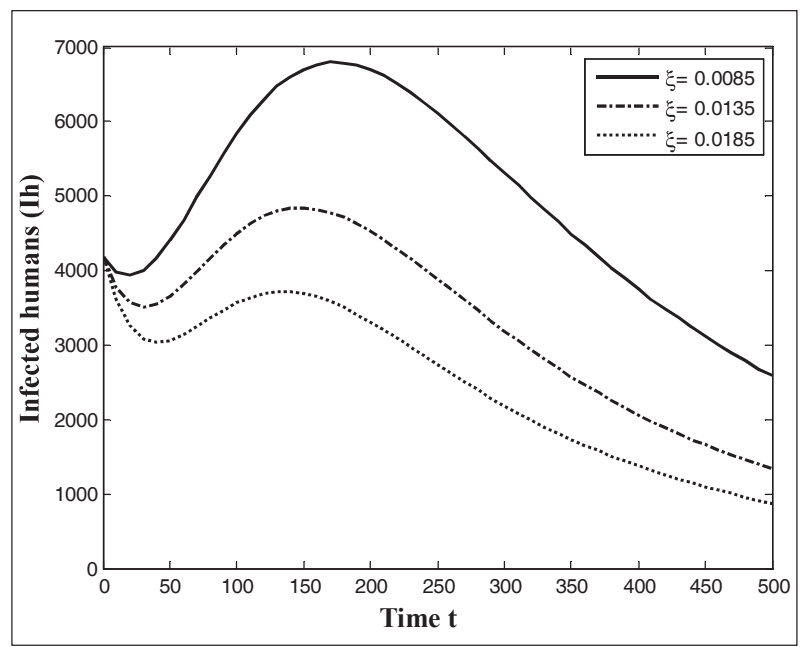

Figure 3: Variation in infective human population $I_{h}$ with time for various values of recovery rate $\xi$, when the transmission coefficient for humans is a constant

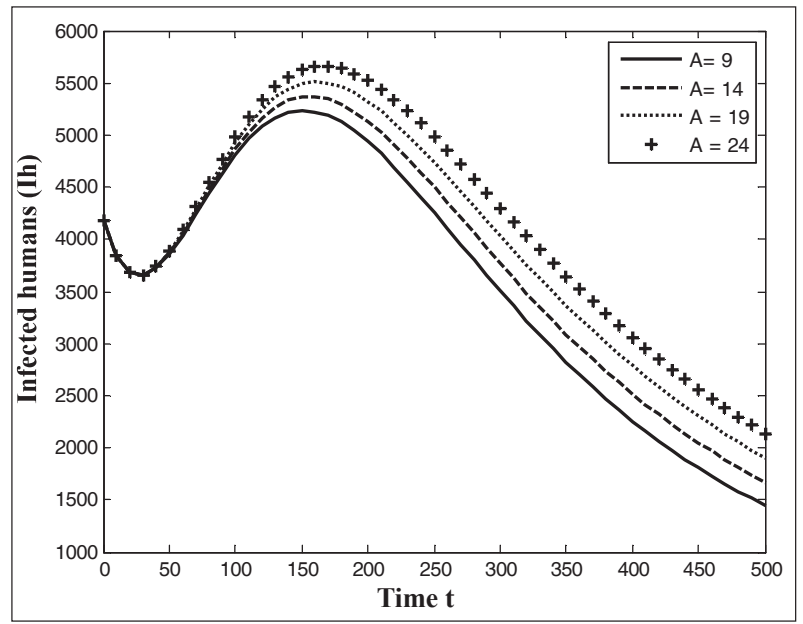

Figure 4: Variation in infective human population $I_{h}$ with time for various values of immigration constant $A$, when the transmission coefficient for humans is a constant

which are based on Gosh et al. (2005) and Hazarika and Bhattacharjee (2011).

The effect of progression rate $\gamma_{h}$, on infected human population $\left(\mathrm{I}_{h}\right)$ is depicted in Figure 2. It is noted that initially when $\gamma_{h}$ is low the infected human population is also less, but as time increases the effect of $\gamma_{h}$ on $I_{h}$ is reversed.

Figure 3 , shows that if recovery rate $\xi$, increases than the $I_{h}$ decreases.

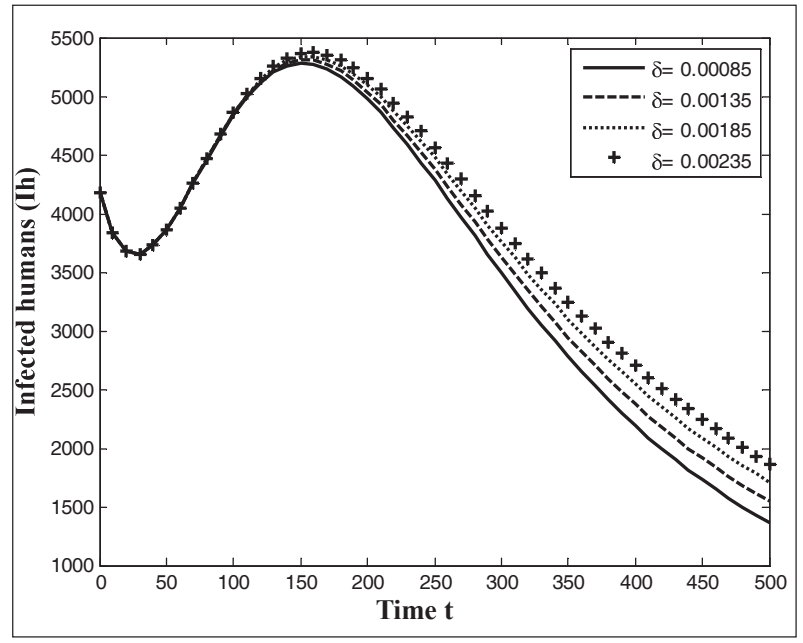

Figure 5: Variation in infective human population $I_{h}$ with time for various values of the rate of immunity loss of recovered humans $\delta$, when the transmission coefficient for humans is a constant 


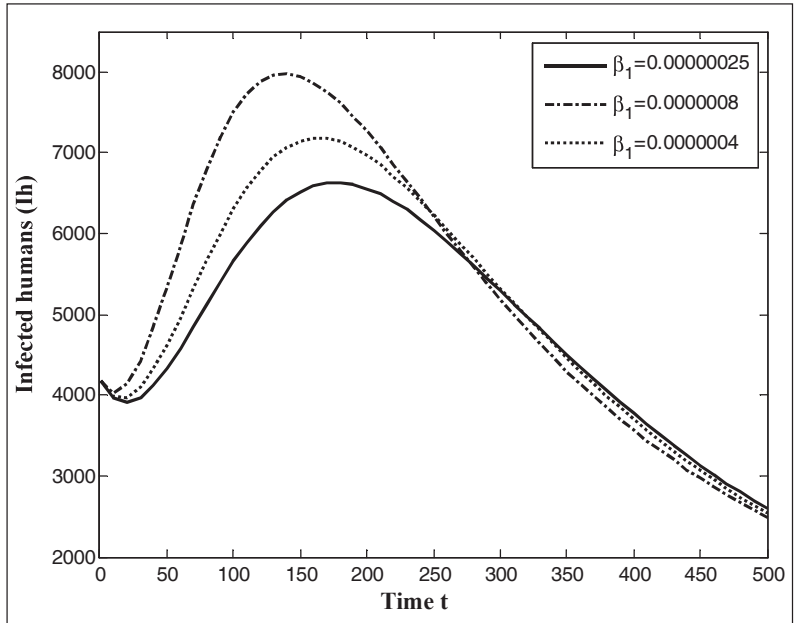

Figure 6: Variation in infective human population $\mathrm{I}_{\mathrm{h}}$ with time for various values of the interaction coefficient $\beta_{1}$, between susceptible humans and exposed mosquitoes, when the transmission coefficient for humans is a constant

Effect of immigration constant ' $A$ ' on $I_{h}$ is shown in figure 4. Infected human population $I_{h}$ increases with the increase in ' $A$ '. $I_{h}$ increases with the increase in rate of immunity loss of recovered humans $\delta$, which is illustrated in Figure 5.

In Figure $6, I_{h}$ is plotted against the various values of $\beta_{1}$ which is the interaction coefficient between

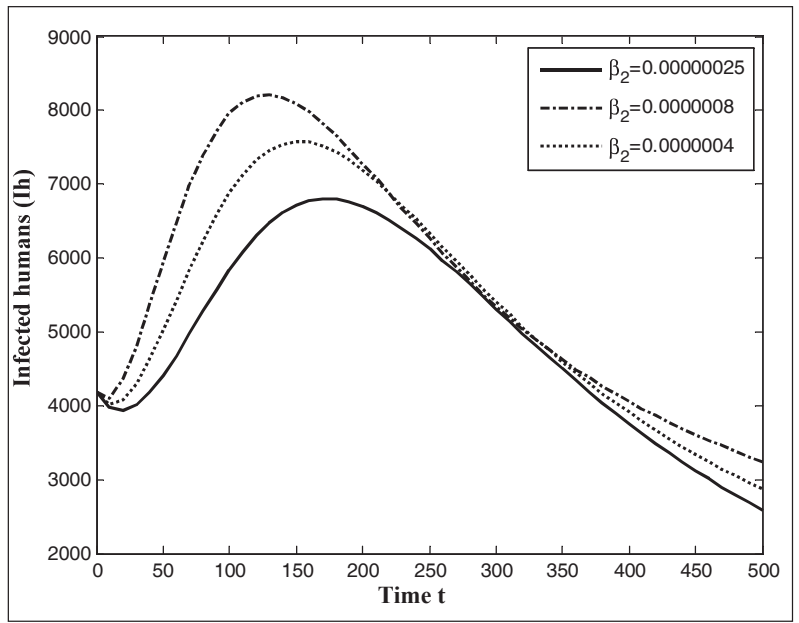

Figure 7: Variation in infective human population $I_{h}$ with time for various values of the interaction coefficient $\beta_{2}$, between susceptible humans and infective mosquitoes, when the transmission coefficient for humans is a constant susceptible humans and exposed mosquitoes. Increase in $\beta_{1}$, increases the $\mathrm{I}_{\mathrm{h}}$. Whereas in Figure 7, $\mathrm{I}_{\mathrm{h}}$ is plotted against the various values of $\beta_{2}$ which is the interaction coefficient between susceptible humans and infected mosquitoes. $\beta_{2}$ also follows the same trend as $\beta_{1}$ with respect to $I_{h}$.

The time-dependent patterns of infection and immunity are illustrated in Figure 8. With the increase in time, first the infected human population increases

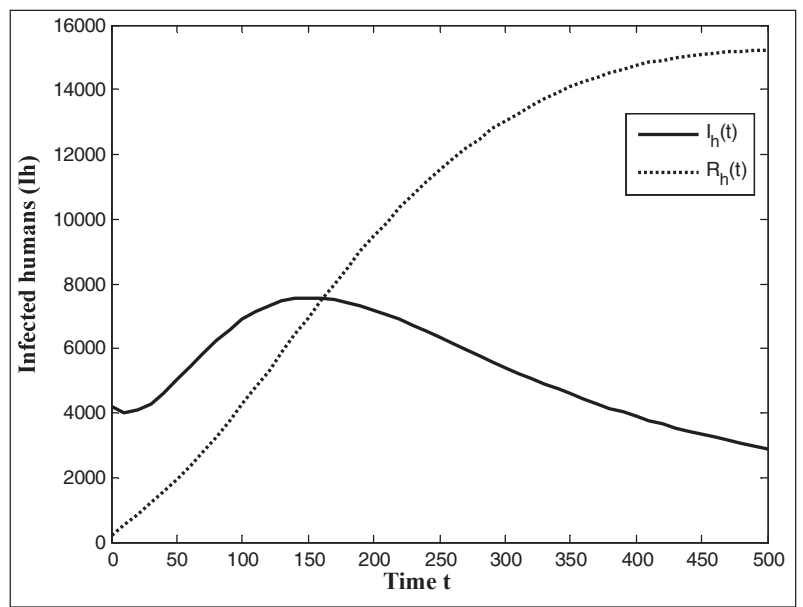

Figure 8: Variation in infective population and recovered population of humans with time when the transmission coefficient for humans is a constant.

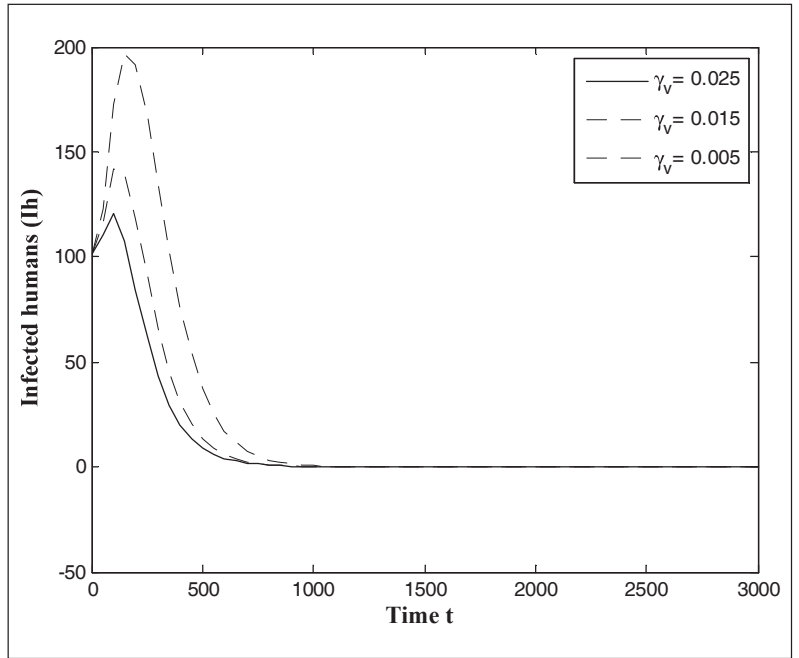

Figure 9: Variation in infective human population $I_{h}$ with time for various values of progression rate $\gamma_{v}$, when the transmission coefficient for humans is a variable. 
and in the gradually decreases, due to the presence of non-linearity terms in the developed model. On the other hand, the proportion of recovered humans increases because of the fact that when some medicinal treatment is given to the infected human, its recovery rate grows significantly.

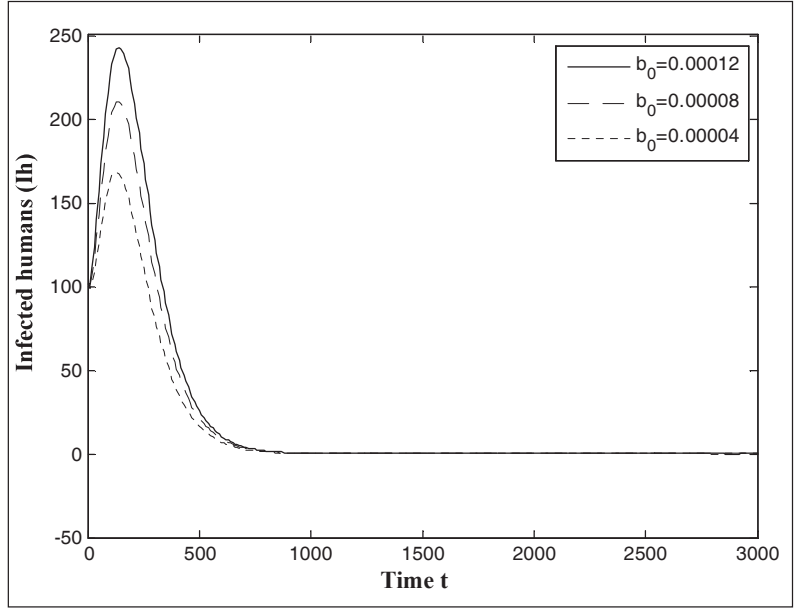

Figure 10: Variation in infective human population $I_{h}$ with time for various values of $b_{0}$, when the transmission coefficient for humans is a variable

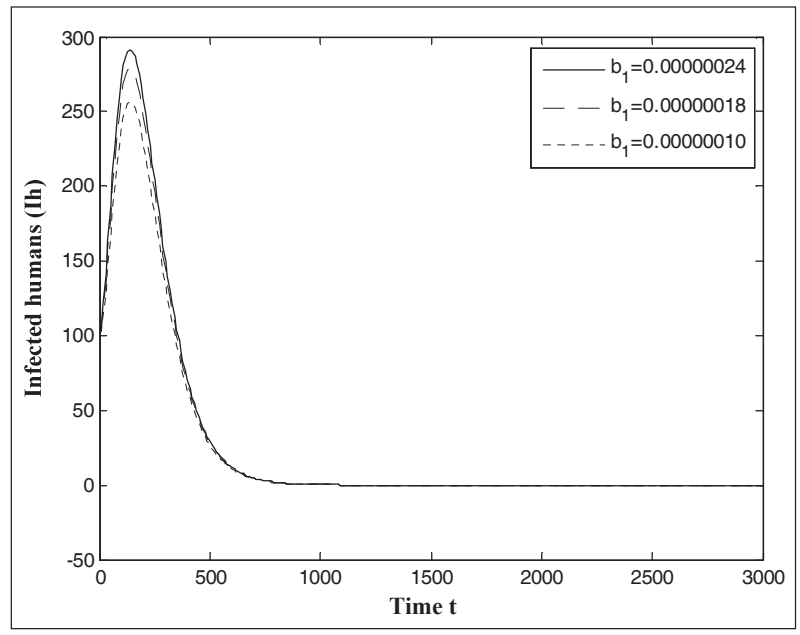

Figure 11: Variation in infective human population $I_{h}$ with time for various values of $b_{1}$, when the transmission coefficient for humans is a variable

Analysis of case 2, where we considered that $\beta_{1}=\mathrm{b}_{0}+$ $\mathrm{b}_{1} \mathrm{~N}_{\mathrm{v}}$ is shown in Figures 9 to 12 . In Figure 9 it is observed that if $\gamma_{\mathrm{v}}$ decreases the $I_{\mathrm{h}}$ increases.

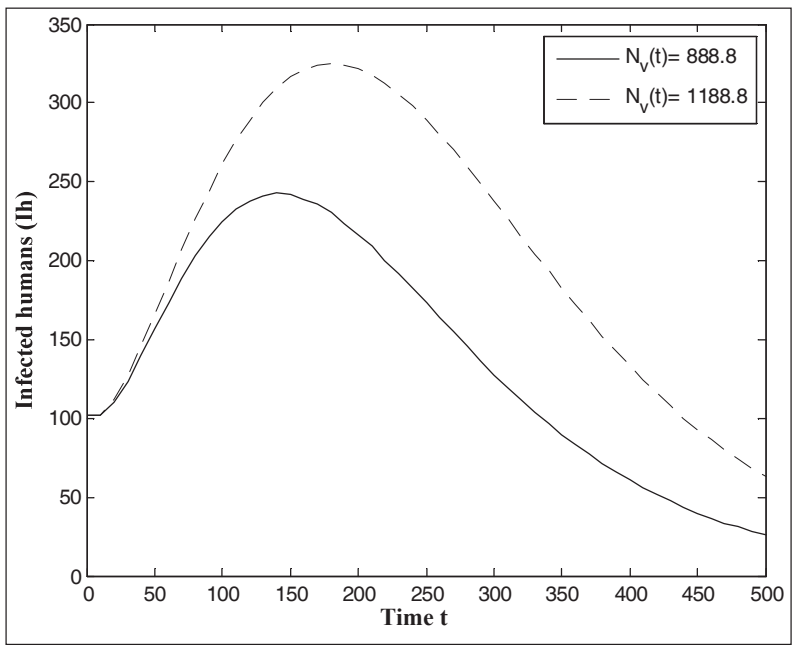

Figure 12: Variation in infective human population $I_{h}$ with time for various values of $N_{v}$, when the transmission coefficient for humans is a variable

Figures 10 and 11, show the variation in $I_{h}$ with respect to $\mathrm{b}_{0}$ and $\mathrm{b}_{1}$. It is depicted from these figures that the infected population of humans increases with the increase in $b_{0}$ and $b_{1}$. Variation in $I_{h}$ for two values of $N_{v}(t)(888.8$, 1188.8 ) is given in Figure 12. It is noted that if $\mathrm{N}_{\mathrm{v}}$ is high then $I_{h}$ is also high.

\section{CONCLUSION}

In this paper, a SEIR model for malaria epidemic is proposed by considering both human and mosquito populations. The exposed class of both human and mosquito populations were included. The major factor which is responsible for the spread of malaria is the transmission coefficient between the human population and the infected mosquito. Numerical results revealed that if the immigration constant ' $A$ ' and human population with immunity loss $\delta$ increases, then the number of infected humans increases, which are clearly illustrated in Figures 4 and 5. It has been concluded that effective control strategies against the malaria vector play an important role in the outburst of the disease. Proper drainage systems and insecticides can lead to elimination of mosquito population.

\section{REFERENCES}

Anderson R.M. \& May R.M. (1991). Infectious Diseases of Humans. Oxford University Press. Oxford, UK.

Bailey N.T.J. (1957). The Mathematical Theory of Epidemics, pp. 134-145. Griffin Publishers, London, UK. 
Cai L., Li. X., Tuncer N., Martcheva M. \& Lashari A. A. (2017). Optimal control of a malaria model with asymptomatic class and super infection. Mathematical Biosciences 288: 94-108.

DOI: https://doi.org/10.1016/j.mbs.2017.03.003

Chamchod F. \& Britton N.F. (2011). Analysis of a vector-bias model on malaria transmission. Bulletin of Mathematical Biology 73(3): 639-657.

DOI: https://doi.org/10.1007/s11538-010-9545-0

Chaves F.L., Kaneko A., Taleo G., Pascual M. \& Wilson L.M. (2008). Malaria transmission pattern resilience to climatic variability is by insecticide-treated nets. Malaria Journal 7(100): 1-14.

DOI: https://doi.org/10.1186/1475-2875-7-100

Chitnis N., Cushing J.M. \& Hyman J.M. (2006). Bifurcation analysis of a mathematical model for malaria transmission. SIAM Journal on Applied Mathematics 67(1): 24-45. DOI: https://doi.org/10.1137/050638941

Chitnis N., Hardy D. \& Smith T. (2012). A periodically-forced mathematical model for the seasonal dynamics of malaria in mosquitoes. Bulletin of Mathematical Biology 74(5): 1098-1124.

DOI: https://doi.org/10.1007/s11538-011-9710-0

Erin M., Thomas S., Smith A. \& Chitnis N. (2013). Estimating malaria transmission through mathematical models. Trends in Parasitology 29(10): 477-482.

Fatmawati \& Tasman H. (2015). An optimal control strategy to reduce the spread of malaria resistance. Mathematical Biosciences 262: 73-79.

DOI: https://doi.org/10.1016/j.mbs.2014.12.005

Forouzannia F. \& Gumel A. (2015). Dynamics of an agestructured two-strain model for malaria transmission. Applied Mathematics and Computation 250(1): 860-886. DOI: https://doi.org/10.1016/j.amc.2014.09.117

Ghosh S., Waite L.J., Clayton H.D. \& Adler R.F. (2014). Can antibodies against flies alter malaria transmission in birds by changing vector behaviour. Journal of Theoretical Biology 358: 93-101.

DOI: https://doi.org/10.1016/j.jtbi.2014.05.020

Gosh M., Chandra P., Sinha P. \& Shukla J.B. (2005). Modeling the spread of bacterial disease: Effect of service providers from an environmentally degraded region. Applied Mathematics and Computation 160: 615-647.

DOI: https://doi.org/10.1016/j.amc.2003.11.022

Gupta S., Swinton J. \& Anderson R.M. (1994). Theoretical studies of the effects of heterogeneity in the parasite population on the transmission dynamics of malaria. Proceedings of the Royal Society B 256(1347): 231-238. DOI: https://doi.org/10.1098/rspb.1994.0075

Hazarika G.C. \& Bhattacharjee A. (2011). Analysis of a malaria model with time mosquito-dependent transmission coefficient for human. Mathematical Sciences 121(1): 93109.

Hethcote H.W. (2000). The mathematics of infectious diseases. SIAM Review 42(4): 599-653.

Koella J.C. (1991). On the use of mathematical models of malaria transmission. Acta Tropica 49: 1-25. DOI: https://doi.org/10.1016/0001-706X(91)90026-G

Li J. (2011). Malaria model with stage-structured mosquitoes. Mathematical Biosciences and Engineering 8(3): 753-768. DOI: https://doi.org/10.3934/mbe.2011.8.753

Ngwa G.A. (2004). Modelling the dynamics of endemic malaria in growing populations. Discrete and Continuous Dynamical Systems B 4(4): 1173-1202. DOI: https://doi.org/10.3934/dcdsb.2004.4.1173

Ngwa G.A. \& Shu W.S. (2000). A mathematical model for endemic malaria with variable human and mosquito populations. Mathematical and Computer Modelling 32(7-8): 747-763. DOI: https://doi.org/10.1016/S08957177(00)00169-2

Ross R. (1916). An application of the theory of probabilities to the study of a priori pathometry. Proceedings of the Royal Society of London, Series A 92(638): 204-230.

Ruan S., Xiao D. \& Beier J. C. (2008). On the delayed RossMacdonald model for malaria transmission. Bulletin of Mathematical Biology 70(4): 1098-1114. DOI: https://doi.org/10.1007/s11538-007-9292-z

Singh S., Shukla J.B. \& Chandra P. (2005). Modelling and spread of malaria: environmental and ecological effects. Journal of Biological Systems 13(1): 1-11.

Wang X., Tang S. \& Cheke R. A. (2016). A stage structured mosquito model incorporating effects of precipitation and daily temperature fluctuations. Journal of Theoretical Biology 411(21): 27-36. DOI: http://dx.doi.org/10.1016/j.jtbi.2016.09.015

World Health Organization (2013). Fact sheet. World Malaria Report- 2013. World Health Organization, Geneva, Switzerland.

Xiao Y. \& Zou X. (2013). Can multiple malaria species copersist? SIAM Journal on Applied Mathematics 73(1): 351-373. 